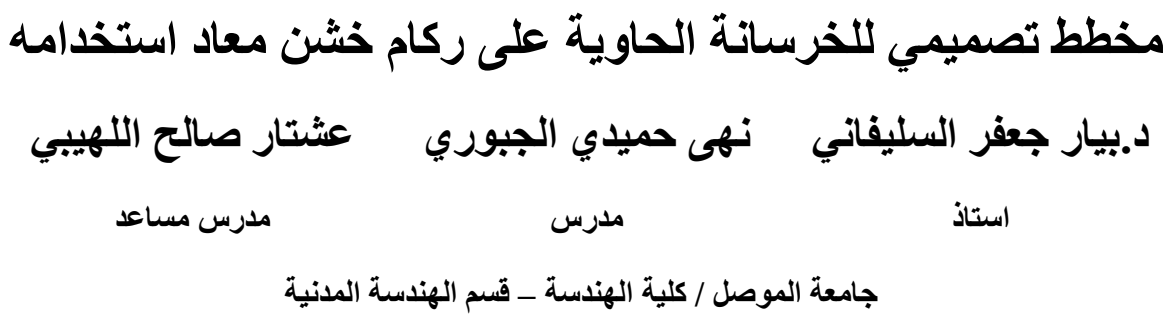

الخلاصة

إن أعادة استخدام مخلفات البناء ثانية يعتبر من النقاط المهمة من اجل الحفاظ على المصادر الطبيعية والتقليل

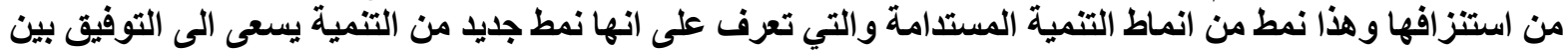

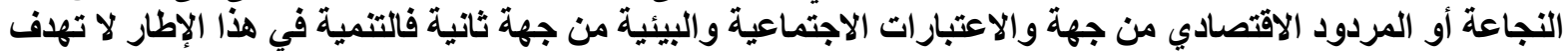

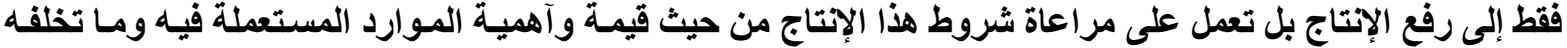

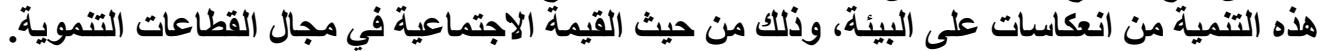

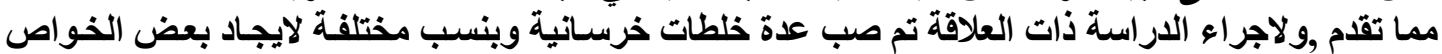

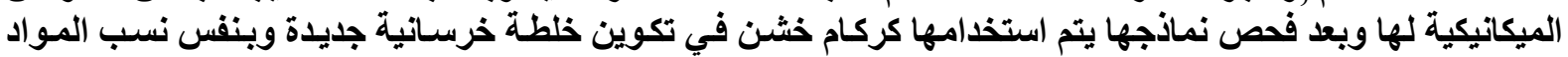

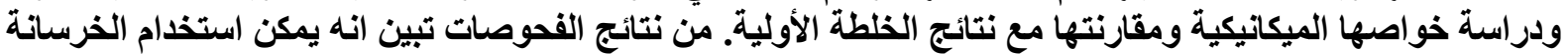

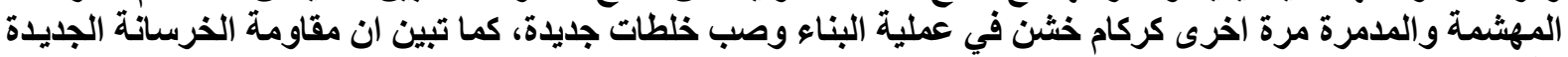

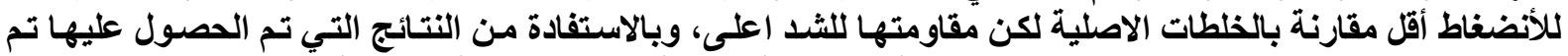

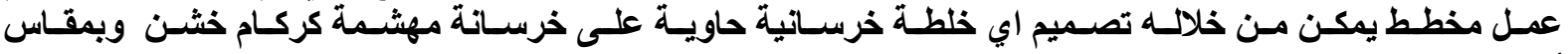
أقصى(25mm).

\title{
Design Chart of Concrete Containing Recycled Coarse Aggregate
}

\author{
Dr. Bayar J. Al-Sulayfani \\ Professor \\ Nuha H. Aljubory \\ Lecturer \\ Ashtar S. Al-Luhybi \\ Assistant Lecturer
}

\begin{abstract}
The re-use of the demolished parts of buildings is considered as an important factor of conserving natural sources and minimizing its consumption, this may be taken as a good development pattern, which leads to compatibility between economical savings and an environmental and social consideration.

Development in this sense not only aims at improving production but also takes into account the abiding by the production rules in considering the available sources, its cost, and its overall effect on environmental and social values. To carry out this research work a number of concrete mixes were prepared using different ratios the specimen then tested the outcome of the crushed concrete specimens were then used as coarse aggregate to constitute new concrete mixes of similar ratios for studying the mechanical properties of those new mixes and comparing then with the original mixes.

The results indicated clearly the possibilities of using recycled destroyed concrete as aggregates also the results showed a reduction in the compressive strength and an increase in tensile strength. Finally a mix design procedure is given foe the future design of any mix using crushed recycled concrete as an aggregate.
\end{abstract}

Key words: Concrete, Recycled aggregate, Mechanical Properties, Design Chart 
أن اعادة استخدام الخرسانة المدمرة او المهيمة كركام معاد في الخرسانة الجديدة عملية مفيدة من ناحية حفظ

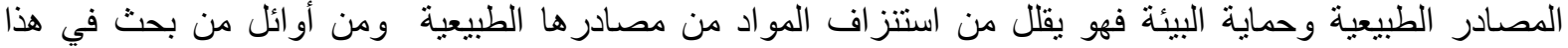

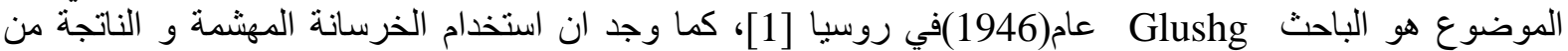

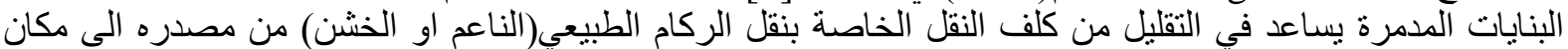

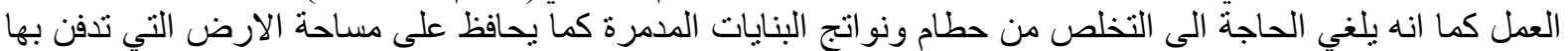

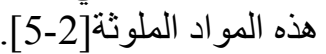
هناك من استفاد من الخرسانة المهشمة في استخدامها كركام ناعج(fine aggregate) وهذا ما قام قام به الباحثان وKalaiarasu and Subramanian

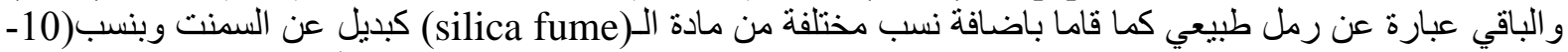

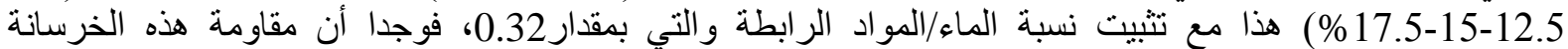
للحو امض و الكلور ايدات تكون أعلى ما يمكن عندما تكون نسبة الـالـو (15\%) (silica fume).

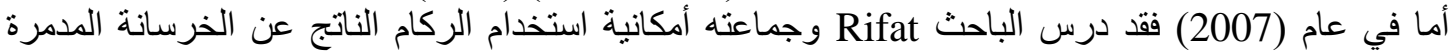

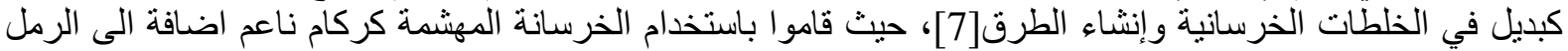

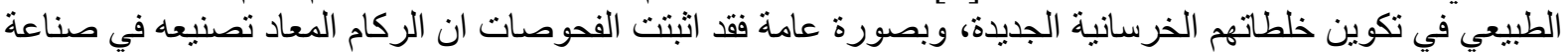
الخرسانة وطبقة الاساس في الطرق يعطي نتائج مقبولة.

أهمية البحث

نظر اً لأهمية هذا الموضوع وجد من الافضل در اسة خو اص الخرسانة الحاوية على ركام خشن ناتج من خرسانة

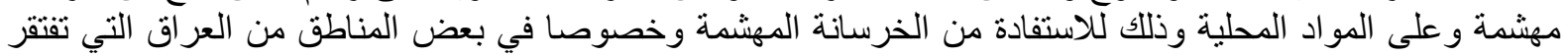

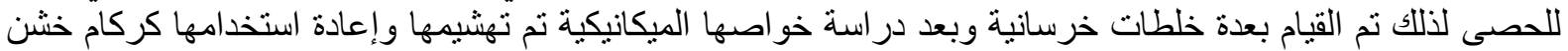

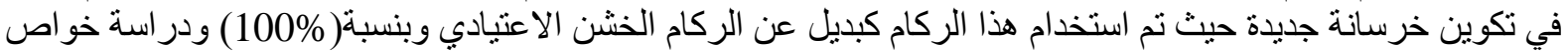

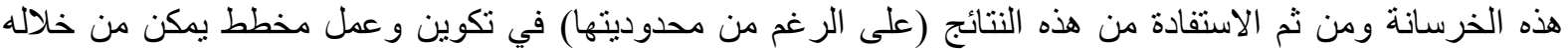
تصميم اي خلطة خرسانية ذات ركام معاد استخدامه وبمقاس اقصى التصى(25mm).

\section{البرنامج العملي}

تم تنفيذ الدر اسة على ثلاثة مر احل

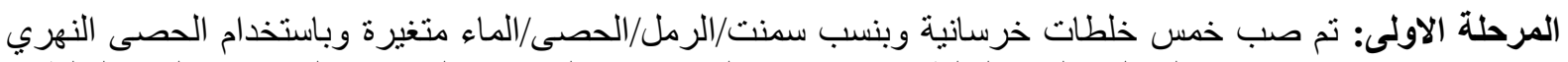

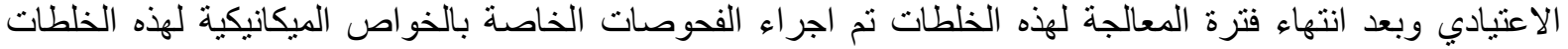
وتشمل مقاومة الانضغاط(مكعب، اسطو انة)، مقاومة الثند، مقاومة الانثناء، ومن ثم تثثيث النتائج.

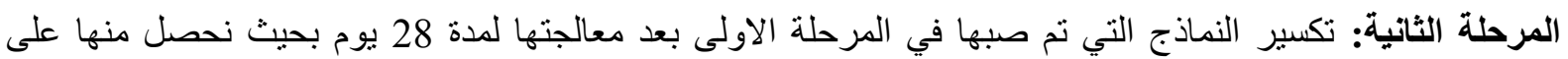

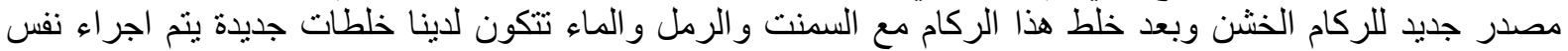

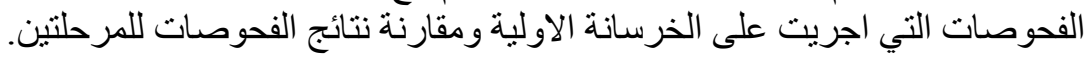

المرحلة الثالثة: عمل مخطط تصميمي للخرسانة الحاوية على ركام خشن ناتج عن خرسانة مهشمة وبمقاس اقصى(25mm) وذللك لتبسيط عملية التصميم هذا النوع من الخرسانة.

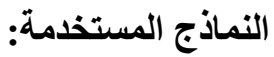

فحص مقاومة الأنضغاط: لاجراء هذا الفحص تم استخدام مكعبات قياسية (150×150×150mm) كما

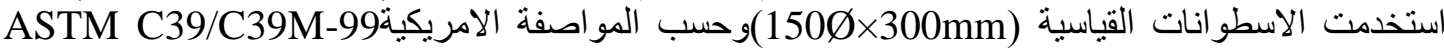

فحص الانشطار: لاجر اء هذا الفحص تم استخدام اسطوانات قياسية (150Ø×300mm) وحسب المواصفة

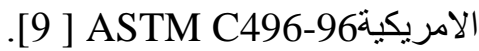




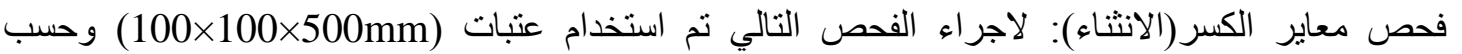

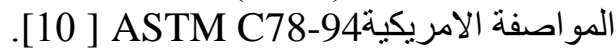

المواد المستخدمة في الخلطة الخرسانية:

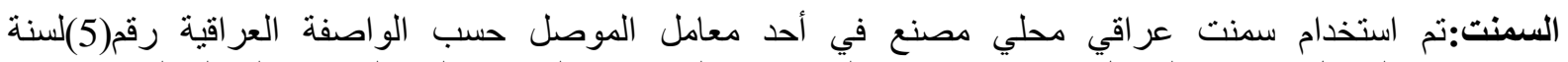
[11984]. الجدول رقم(1)و الجدول رقم(2)يوضحان الخصائص الفيزيائية و الكيميائية لهذا السمنت على التو الي.

\section{جدول (1) التحليل الكيميائي للسمنت المستخدم ومركباته الرئيسية}

\begin{tabular}{|c|c|c|}
\hline Property & Test result (Percentage) & Standard IQS, No.5 \\
\hline 1.Oxides composition: & \multicolumn{2}{|l|}{} \\
\hline Alumina, $\mathrm{Al}_{2} \mathrm{O}_{3}$ & 4.7 & \\
\hline Silica, $\mathrm{SiO}_{2}$ & 21.5 & \\
\hline Ferric Oxide, $\mathrm{Fe}_{2} \mathrm{O}_{3}$ & 2.41 & \\
\hline Lime, $\mathrm{CaO}$ & 62.86 & Max. 2.8 \\
\hline $\begin{array}{c}\text { Sulphuric } \mathrm{Anhydride}, \\
\mathrm{SO}_{3}\end{array}$ & 2.7 & Max. 4 \\
\hline Magnesia, $\mathrm{MgO}$ & 2.25 & \\
\hline 2.Compounds composition: & & \\
\hline $\mathrm{C}_{3} \mathrm{~S}$ & 46.14 & \\
\hline $\mathrm{C}_{2} \mathrm{~S}$ & 27.18 & \\
\hline $\mathrm{C}_{3} \mathrm{~A}$ & 8.4 & \\
\hline $\mathrm{C}_{4} \mathrm{AF}$ & 7.3 & \\
\hline
\end{tabular}

جدول (2) الخصائص الفيزيائية للسمنت المستخدم.

\begin{tabular}{|c|c|c|}
\hline Property & Test result & $\begin{array}{c}\text { Standard IQS, } \\
\text { No.5 }\end{array}$ \\
\hline Fineness(Residue on B.S. sieve No. 170) & $8 \%$ & Max. 10\% \\
\hline Specific surface "Blaine" & $3358.5\left(\mathrm{~cm}^{2} / \mathrm{gm}\right)$ & $\begin{array}{c}\text { Min. } \\
2250\left(\mathrm{~cm}^{2} / \mathrm{gm}\right)\end{array}$ \\
\hline Initial setting time & $120(\mathrm{~min})$. & $\geq 45(\mathrm{~min})$. \\
\hline Final setting time & $200(\mathrm{~min})$. & $\leq 600(\mathrm{~min})$. \\
\hline Specific gravity & 3.15 & \\
\hline $\begin{array}{c}\text { Compressive strength } \\
70.7 m m \text { cubes }\end{array}$ & & $\geq 15.0(\mathrm{MPa})$ \\
\hline at 3 days & $18.0(\mathrm{MPa})$ & $\geq 23.0(\mathrm{MPa})$ \\
\hline at 7 days & $25.0(\mathrm{MPa})$ & \\
\hline
\end{tabular}

الرمل:تم استخدام رمل من نوع منوسط النعومة ومطابق للمواصفات البريطانية(B.S.882:1992)] [12]، الجدول رقم(3)

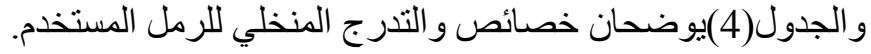




\begin{tabular}{|c|c|c|c|}
\hline \multicolumn{4}{|c|}{ جدول (3) خصائص الركام الناعم } \\
\hline $\begin{array}{l}\text { Type of } \\
\text { fine } \\
\text { aggregate }\end{array}$ & Color & $\begin{array}{l}\text { Specific } \\
\text { Gravity }\end{array}$ & $\begin{array}{c}\text { Absorption } \\
\%\end{array}$ \\
\hline \multirow{2}{*}{$\begin{array}{l}\text { Medium } \\
\text { sand }\end{array}$} & \multirow{2}{*}{ Brown } & S.S.D basis & \multirow{2}{*}{2.0} \\
\hline & & 2.56 & \\
\hline
\end{tabular}

\begin{tabular}{|c|c|c|}
\hline \multicolumn{3}{|c|}{ جذول (4) تدرج الركام الناعم } \\
\hline Sieve Size & $\begin{array}{c}\text { \%Specification Limits } \\
\text { According to } \\
\text { B.S.882:1992 }\end{array}$ & $\begin{array}{c}\text { \% Passing of sample to be } \\
\text { used }\end{array}$ \\
\hline No.4 & $89-100$ & 100 \\
\hline No.8 & $60-100$ & 80 \\
\hline No.16 & $30-100$ & 67 \\
\hline No.30 & $15-100$ & 44 \\
\hline No.50 & $5-70$ & 14 \\
\hline No.100 & $0-15$ & 3 \\
\hline
\end{tabular}

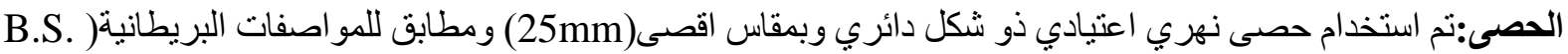

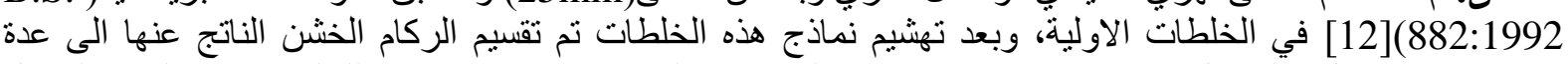

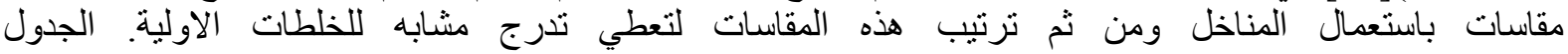
رقم(5)و الجدول(6)يوضحان خصائص والتدرج المنخلي للحصى الاعتيادي وللركام الخشن المعاد استخدامه.

\begin{tabular}{|c|c|c|}
\hline \multicolumn{3}{|c|}{ جدول (5) تدرج الركام الخشن } \\
\hline Sieve Size & $\begin{array}{c}\text { \%Specification Limits } \\
\text { According to } \\
\text { B.S.882:1992 }\end{array}$ & $\begin{array}{c}\text { \% Passing of sample to } \\
\text { be used }\end{array}$ \\
\hline $11 / 2$ in. & 100 & 100 \\
\hline 1 in. & $95-100$ & 97.5 \\
\hline $1 / 2$ in. & $25-60$ & 42.5 \\
\hline No.4 & $0-10$ & 5 \\
\hline No.8 & $0-5$ & 0 \\
\hline No.16 & 0 & 0 \\
\hline
\end{tabular}

\begin{tabular}{|c|c|c|c|c|}
\hline $\begin{array}{c}\text { Type of coarse } \\
\text { aggregate }\end{array}$ & $\begin{array}{c}\text { Maximum } \\
\text { aggregate } \\
\text { size (mm) }\end{array}$ & $\begin{array}{c}\text { Specific } \\
\text { Gravity S.S.D }\end{array}$ & $\begin{array}{c}\text { Angularity } \\
\text { Index }\end{array}$ & $\begin{array}{c}\text { Absorptio } \\
\text { n\% }\end{array}$ \\
\hline $\begin{array}{c}\text { Rounded } \\
\text { gravel }\end{array}$ & 25 & 2.69 & 1.3 & 0.35 \\
\hline $\begin{array}{c}\text { Recycled } \\
\text { aggregate }\end{array}$ & 25 & 2.46 & 2.57 & 3.57 \\
\hline
\end{tabular}


الماء:تم استخدام ماء الثرب الاعتيادي في جميع الخلطات الخرسانية وكذللك لاغر اض المعالجة.

تم صب الخلطات الخرسانية بو اسطة الخلاطة الكهربائية واستعملت الهزاز ات(Vibrators)الكهربائية في عملية

رص الخرسانة.

النتائج

الجدول رقم(7) و الجدول رقم (8) يبينان الخلطات الخرسانية المصممة وفق مقاومات انضغاط مختلفة اضافة الى خلطنين

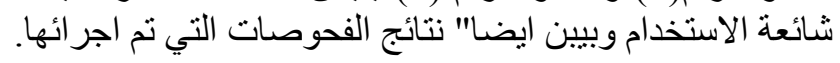

جدول (7) نسب الخلطات والخواص الميكاتيكية للخرسانة الاعتيادية

\begin{tabular}{|c|c|c|c|c|c|c|}
\hline $\begin{array}{c}\text { Mix } \\
\text { proportion }\end{array}$ & w/c* & $\begin{array}{c}\text { Slump } \\
(\mathrm{mm})\end{array}$ & $\begin{array}{c}\text { Compressive } \\
\text { strength of } \\
\text { cylinder } \\
(\mathrm{MPa})^{* *}\end{array}$ & $\begin{array}{c}\text { Compressive } \\
\text { strength of } \\
\text { cube(MPa)** }\end{array}$ & $\begin{array}{c}\text { Splitting } \\
\text { strength } \\
(\mathrm{MPa})^{* *}\end{array}$ & $\begin{array}{c}\text { Flexural } \\
\text { strength } \\
\text { (modulus } \\
\text { of rupture) } \\
\text { (MPa)** }\end{array}$ \\
\hline $1: 1.9: 2.85$ & 0.35 & 90 & 29.6 & 45.5 & 2.9 & 6.3 \\
\hline $1: 2.3: 3.15$ & 0.40 & 85 & 26.3 & 42.4 & 2.8 & 5.9 \\
\hline $1: 2: 4$ & 0.44 & 85 & 24.4 & 38.5 & 2.7 & 5.7 \\
\hline $1: 3.15: 3.8$ & 0.47 & 80 & 21.2 & 36.4 & 2.6 & 5.4 \\
\hline $1: 3: 6$ & 0.59 & 70 & 18.4 & 31.1 & 2.5 & 4.4 \\
\hline
\end{tabular}

$*_{\mathrm{w}} / \mathrm{c}:$ water to cement ratio

**tested at 28 day

جذول (8) نسب الخلطات والخواص الميكانيكية للخرسانة المعاد استخدامها

\begin{tabular}{|c|c|c|c|c|c|c|}
\hline $\begin{array}{c}\text { Mix } \\
\text { proportion }\end{array}$ & w/c & $\begin{array}{c}\text { Slump } \\
(\mathrm{mm})\end{array}$ & $\begin{array}{c}\text { Compressiv } \\
\text { e strength } \\
\text { of cylinder } \\
(\mathrm{MPa})\end{array}$ & $\begin{array}{c}\text { Compressive } \\
\text { strength of } \\
\text { cube(MPa) }\end{array}$ & $\begin{array}{c}\text { Splitting } \\
\text { strength } \\
(\mathrm{MPa})\end{array}$ & $\begin{array}{c}\text { Flexural } \\
\text { strength } \\
\text { (modulus of } \\
\text { rupture) } \\
\text { (MPa) }\end{array}$ \\
\hline $1: 1.9: 2.85$ & 0.35 & 70 & 27.2 & 43.0 & 3.6 & 5.7 \\
\hline $1: 2.3: 3.15$ & 0.40 & 80 & 24.3 & 37.6 & 3.5 & 5.5 \\
\hline $1: 2: 4$ & 0.44 & 70 & 21.1 & 35.9 & 3.3 & 5.2 \\
\hline $1: 3.15: 3.8$ & 0.47 & 65 & 18.5 & 31.0 & 3.2 & 5.6 \\
\hline $1: 3: 6$ & 0.59 & 65 & 16.5 & 28.0 & 3.1 & 4.1 \\
\hline
\end{tabular}

مناقثة النتائج

\section{قابلية التشغيل(Workability):}

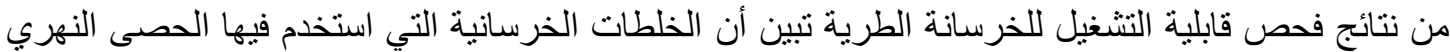

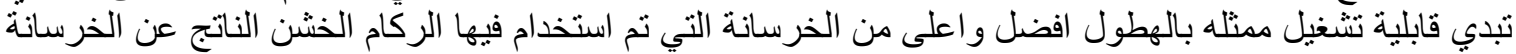

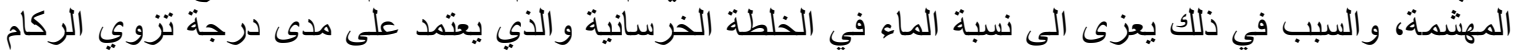

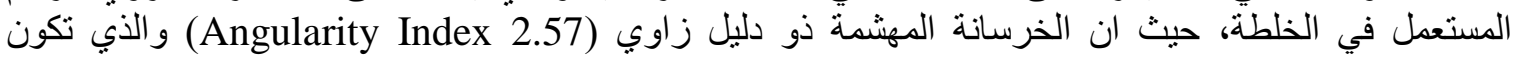

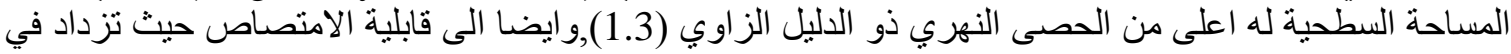

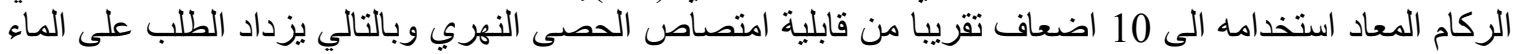




\section{مقاومة الانضغاط(Compressive Strength):}

يوضح الثكلان (1) و(2) العلاقة بين مقاومة الانضغاط للخرسانة مع نسبة الماء/السمنت للاسطوانات

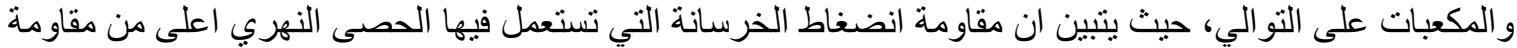
انضغاط الخرسانة المتكونة من الخرسانة المهشمة ولعل السبب في نقص الاخيرة يعود الى في فئل

أـ الخرسانة المتكونة من الخرسانة المهشمة أكثر مسامية (more porous) من الخرسانة المتكونة

من حصى نهري.

بـ مدى مقاومة وتحميل الخرسانة المهثمة أقل من تحمل الحصى النهري لقوى الانضغاط.

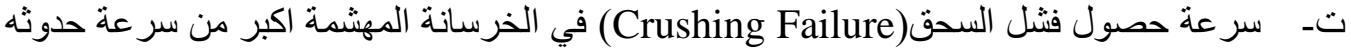

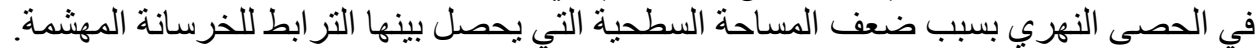

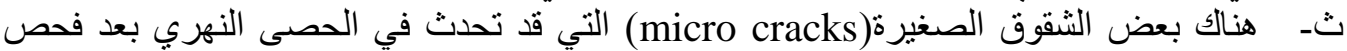

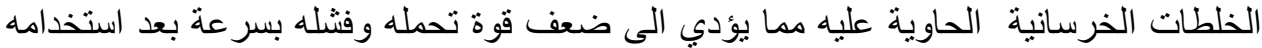
مرة اخرى في الخرسانة الحاوية على خرسانة ملية مهنمة.

مما سبق بالامكان تلخيص بعض خصائص الحصى النهري و الخرسانة المهثمة حيث ان شكل حبييات الخرسانة المهشمة

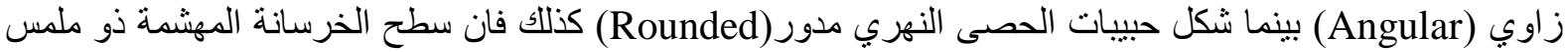

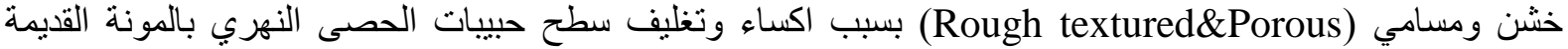

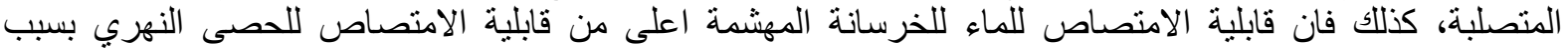

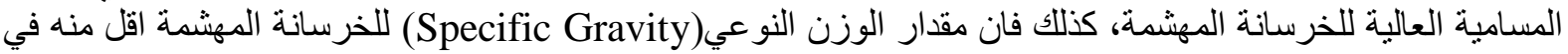
الحصى النهري بسبب المسامية العالية وزيادة نسبة الفجوات في الخرسانة المهيمة عنها في الحصى النهري.

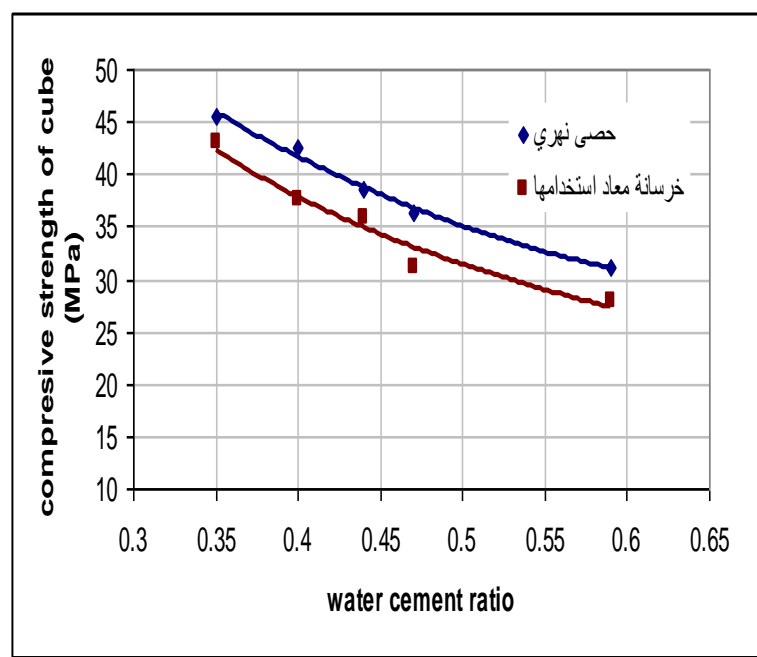

الثكل(2): منحني علاقة مقاومة الانضغاط

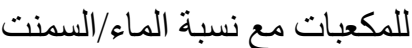

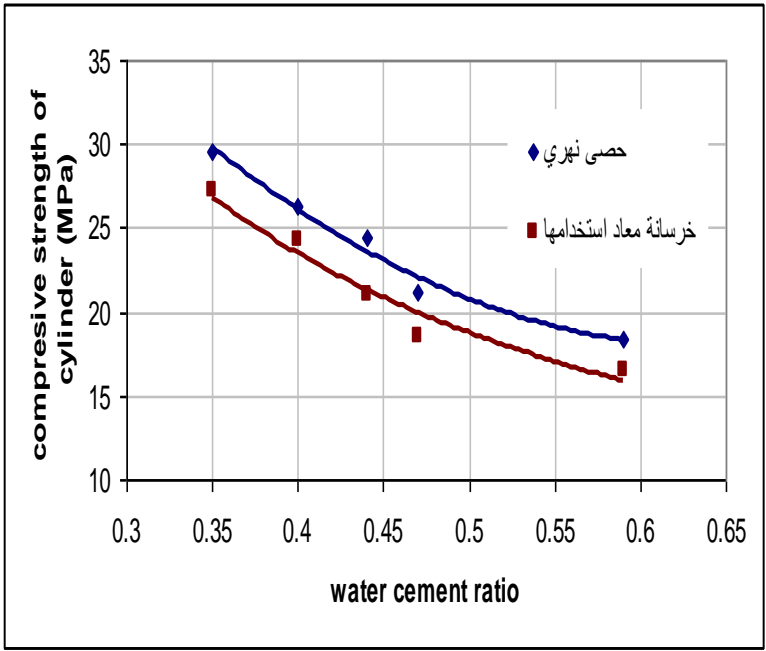

الشكل(1): منحني علاقة مقاومة الانضغاط

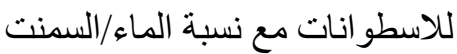

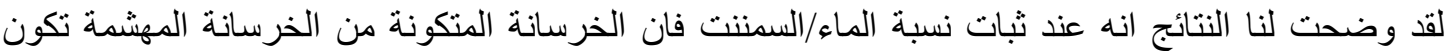
مقاومة الثد لها اكبر من مقاومة شد الخرسانة المتكونة من الحصى النهري النهات الاعتبادي وكما موضح في في الثكل(3). ولعل السبب الرئيسي الذي يعلل هذه الظاهرة يعزى الى قوة التماسك العالية بين عجينة السمنت مع الخرسانة

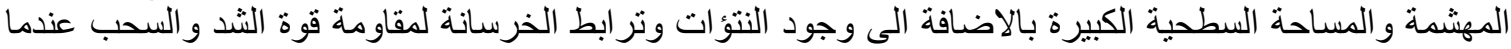
يتعرض لها. 
و هنا تكمن اهمية حسابات الحدية(Angularity) في اختلاف مقاومة الثد في الخرسانة الاعتبادية عنها في الخرسانة المتكونة من الخرسانة المهيمة.

\section{معاير الكسر(الانثتاء)(Modulus of Rupture):}

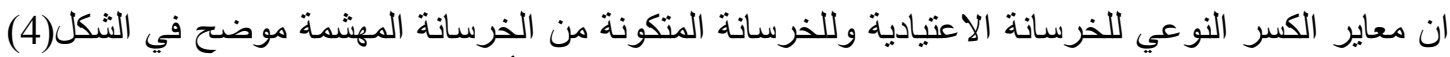

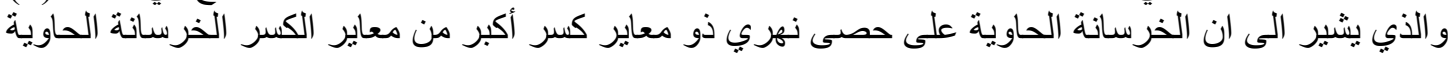
على خرسانة مهثمة.

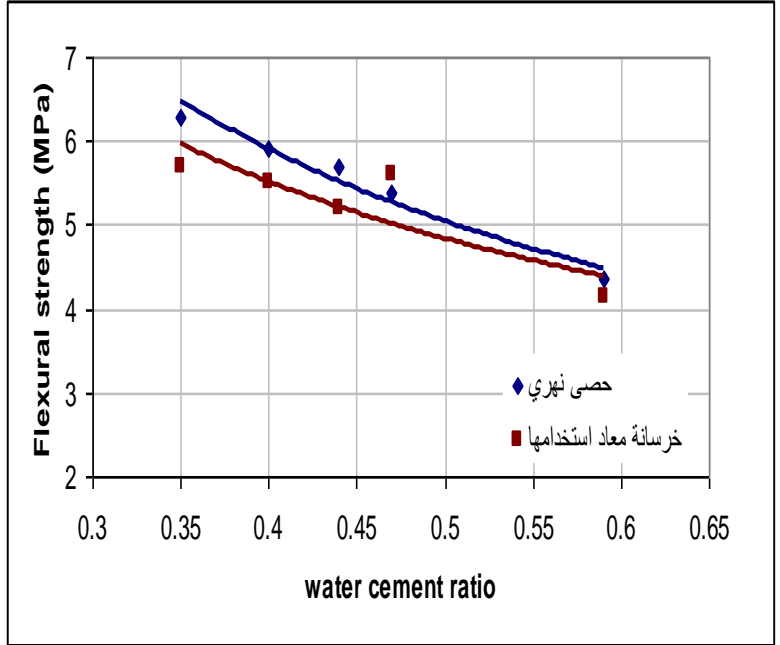

الثكل(4): منحني علاقة معاير الكسر مع نسبة الماء/السمنت

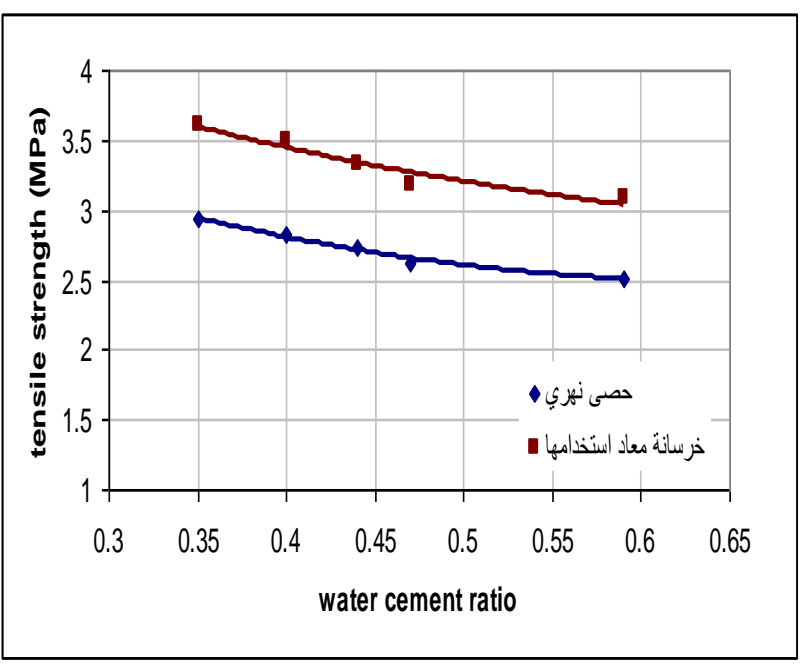

الثكل(3) : منحني علاقة مقاومة الثد مع نسبة الماء/السمنت علأكفة

\section{طريقة لتصميم الخلطات الخرسانية باستعمال الخرسانة المهشمة كمواد خثنة:}

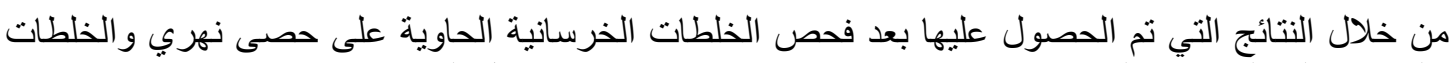
الخرسانية الحاوية على الخرسانة المهشمة كركام خشن ممكن رسم عدة منحنيات تمثل العلاقة بين الخين

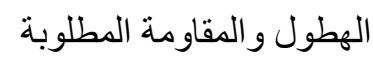

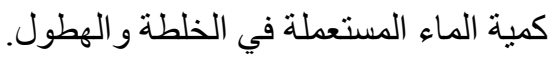
مقاومة الانضغاط وكمية السمنت الو اجب الستخدامة فئه

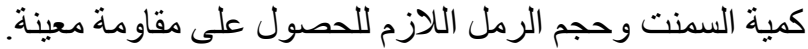
كمية السمنت وحجم الخرسانة المهشمة اللازمة للحصول على على المقاومة المعة المطلوبة. الشكل(5) يبين مخطط يمكن من خلاله تصميم اي خلطة خرسانية حاوية على خرسانة مهشمة كركام خشن وبمقاس اقصى(25mm). مثال توضيحي لاستخدام المخطط: لغرض تصميم خلطة خرسانية حاوية على خرسانة مهثمة بمقاس أقصى(25mm) وبقابلية تشغيل(90mm) وبمقاومة انضغاط(20MPa)

1-نأخذ قابلية تشغيل(90mm) ويقاطع مع خط الهطول وخط مقاومة الانضغاط 2-ومن نقاط التقاطع يتم رسم خطوط أفقية للحصول على كمية السمنت و الرمل و الحصى و الماء 


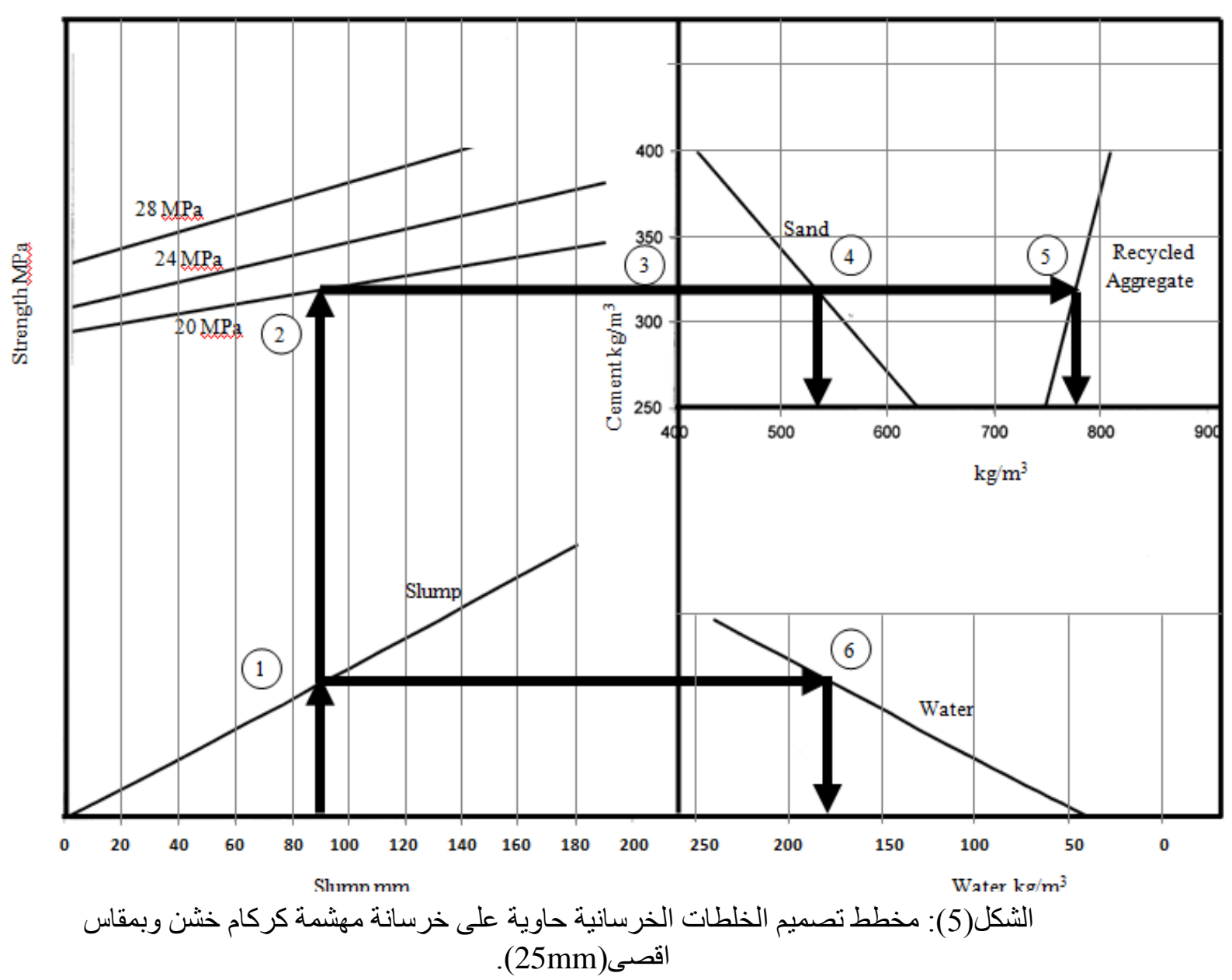

المصادر

1. Gluzhge, P.J., The work of scientific research institute. Gidrotekhnicheskoye Stroitel'stvo 4:27-28. (only available in Russian).

2. Khaldoun Rahal, "Mechanical properties of concrete with recycled coarse aggregate", Building and Environment, Volume 42, Issue 1, January 2007, Pages 407-415

3. Buck. A.D., "Recycled concrete as a source of aggregate", ACI Journal 74 (1977) (5), pp.212-219

4. Hansen, T.C. and Hedegard ,S.E., "Properties of recycled aggregate concrete as affected by admixtures in original concretes", ACI Journal 81 (1984) (1), pp. 21-26.

5. González-Fonteboa, B. and Martínez-Abella. F., "Concretes with aggregates from demolition waste and silica fume. Materials and mechanical properties”, Building and Environment, Volume 43, Issue 4, April 2008, Pages 429-437.

6. Kalaiarasu, S.M, and Subramanian, K., "Properties of Recycled Aggregate Concrete with Silica Fume", Journal of pplied Sciences 6(14) 2006 pp. 2956-2958.

7. Rifat, R., Salah, T., Ali B., and Hani, B., " PROPERTIES OF RECYCLED AGGREGATE IN CONCRETE AND ROAD PAVEMENT APPLICATIONS”, The Islamic University Journal, Vol.15, No. 2,2007, pp 247-264.

8. ASTM, Designation: C 39/C 39M-99, "Standard Test Method for Compressive Strength of Cylindrical Concrete Specimen", Annual Book of ASTM Standards, 
VOL. 04. 02, 5 pp.

9. ASTM, Designation: C469-94, "Standard Test Method for Static Modulus of Elasticity and Poisson's Ratio of Concrete in Compression Loading)", Annual Book of ASTM Standards, VOL. 04. 02, 4 pp.

10. ASTM, Designation: C78-94, "Standard Test Method for Flexural Strength of Concrete(Using Simple Beam with Third-Point Loading)", Annual Book of ASTM Standards, VOL. 04. 02, 3 pp.

$$
\text { 11. المو اصفة القياسية العر اقية رقم (5) لسنة } 1984 \text { (السمنت البورتلاندي). }
$$

12. B.S. 882-1992, "British Standard Specification for Aggregate from Natural Sources for Concrete", 1992. 